\title{
OBSERVING THE NUCLEOSYNTHESIS FROM CORE COLLAPSE SUPERNOVAE
}

\author{
Claes Fransson \\ Stockholm Observatory \\ S-133 00 Saltsjöbaden, Sweden
}

\section{INTRODUCTION}

Since the appearance of the classical papers on stellar nucleosynthesis in the 1950's most of the observational tests have been through indirect sources of information. Even though this has been rather successful (cf. various contributions in this volume), it represents only an average over all sources, yielding little information about specific stars. The most direct evidence comes from observations of young galactic supernova remnants (age less than $\sim 10^{3}$ years). Unfortunately, the analysis of X-ray data are hampered by a lack of understanding of the detailed physics, eg. non-equilibrium and plasma effects, as well as by observational problems (Itoh and Nomoto, 1987). Optical observations (cf. Raymond, 1984) have given some valuable insight of eg. the oxygen-rich remnants. The analysis of these are, however, suffering from the fact that only a small fraction of the mass is seen in the optical.

Although being the most direct approach, comparatively little attention has been paid to direct observations of the nucleosynthesis in supernovae during the first years after the explosion. The main exception has been the modelling of the late Fe/Co spectra of Type I supernovae (Meyerott, 1980; Axelrod 1980; this volume), and the early phases of Type I's by Branch et al. (1985). Both for Type I's and II's the early stages (before $\sim 200$ days) are complicated by NLTE effects, and the non-transparent nature of the envelope. Therefore, the phase most suitable for analysis is the epoch after which the envelope is transparent, and the density low enough to make NLTE effects tractable. The most important condition is that the continua of the excited levels should be optically thin, so that a nebular approximation for the radiative transfer can be used. In this paper I will sketch the basic physics involved in this type of models, and then discuss some applications to Type $\mathrm{Ib}$ and Type II supernovae. For a more detailed account see Fransson and Chevalier (1987, 1988; in the following FC87 and FC88) and Fransson (1987).

\section{RADIOACTIVE EXCITATION}

Both from observations (Barbon et al. 1984) and from theory (eg. Woosley, this volume) there is strong evidence for the formation of $\sim 0.05-0.3 \mathrm{M}_{\odot}$ of ${ }^{56} \mathrm{Ni}$ in the explosion of a massive star. This decays first to ${ }^{56} \mathrm{Co}$ on a time scale of 8.6 days and then to ${ }^{56} \mathrm{Fe}$ on 114 days, emitting $94 \%$ of the energy as $0.5-3 \mathrm{MeV} \gamma$-rays and $4 \%$ in positrons. The trapping and thermalization of these are determined mainly by the density and $\gamma$-ray optical depth of the oxygen core. By the oxygen core I mean all mass inside the oxygen shell, and similarly with the helium core. The important parameters are thus the mass, $\mathrm{M}_{\mathrm{C}}$, and expansion velocity, 
$\mathrm{V}_{C}$, of this. The mass of the oxygen core is relatively well determined, for a $15 \mathrm{M}_{\odot}$ star $\sim 1.1$ $\mathrm{M}_{\odot}$, increasing to $\sim 3.8 \mathrm{M}_{\odot}$ for $25 \mathrm{M}_{\odot}$ (Woosley and Weaver, 1987). The expansion velocity is less certain and depends on the deceleration of the core expansion by the hydrogen envelope. The total mass loss of the progenitor is therefore crucial. For stars more massive than $\sim 20 \mathrm{M}_{\odot}$ mass loss may change the star from a red supergiant with an extended envelope, to a naked WR star (eg. Maeder, this volume). Calculations show that for a $15 \mathrm{M}_{\odot}$ star without mass loss the core velocity is $\sim 900 \mathrm{~km} / \mathrm{s}$ (Weaver and Woosley, 1980). For the same core mass, but without a H-envelope, the velocity increases to $\sim 2500 \mathrm{~km} / \mathrm{s}$ (Woosley and Weaver, 1987). Loss of part of the He-mantle may increase this further. The average density in the oxygen core is $\sim 5.7 \times 10^{8}\left(\mathrm{M}_{\mathrm{C}} / 1 \mathrm{M}_{\odot}\right)\left(\mathrm{V}_{\mathrm{C}} / 10^{3} \mathrm{~km} / \mathrm{s}\right)^{-3} \mathrm{tyr}^{-3} \mathrm{~cm}^{-3}$, and the energy averaged $\gamma$-ray optical depth $\tau_{\gamma}{ }^{2}\left(\mathrm{M}_{\mathrm{c}} / 1 \mathrm{M}_{\odot}\right)\left(\mathrm{V}_{\mathrm{c}} / 10^{3} \mathrm{~km} / \mathrm{s}\right)^{-2} \mathrm{t}_{\mathrm{yr}}{ }^{-2}$. Hydrodynamical calculations show, however, that the density structure is highly non-uniform due to radioactive heating, which creates a bubble in the center, with most of the mass in a thin shell (Woosley and Weaver, 1987; Nomoto et al., 1987). The shell is likely to be subject to the Rayleigh-Taylor instability, smoothing the density distribution and causing a mixing of the elements.

The thermalization of the $\gamma$-rays proceeds in several steps: First the $\gamma$-rays loose their energy by Compton scattering off the bound and free electrons, producing fast nonthermal electrons with energies of $0.1-1 \mathrm{MeV}$. These loose their energy by ionizations, excitations and heating of the thermal, free electrons. In most cases direct excitations are unimportant. The ionizations are balanced by recombinations, and the heating by collisional excitations of low energy levels, mainly in the optical and near-IR. Each absorbed $\gamma$-ray photon thus produces $\sim 10^{6}$ photons in the optical. The thermalization is treated in detail in FC 88, and for an Fe/Co plasma by Axelrod (1980). In this paper I will mainly discuss the application of these models to Type Ib and Type II supernovae, and will therefore only summarize the main ingredients.

The $\gamma$-ray and electron thermalization is modelled by Monte Carlo technique, giving the heating and ionization rates for a given composition and ionization state. This is done iteratively. The recombination and cooling include all important atomic processes. Most of the cooling is done by forbidden and semi-forbidden lines of neutral and singly ionized elements. For the line transfer we use the Sobolev approximation, and for the diffuse continuum a lambda iteration. For $\mathrm{O} I$, and $\mathrm{Ca}$ II we take 9 and 4 levels into account. The elements included are $\mathrm{He}, \mathrm{C}, \mathrm{N}, \mathrm{O}, \mathrm{Ne}, \mathrm{Na}, \mathrm{Mg}, \mathrm{Si}, \mathrm{S}, \mathrm{Ar}, \mathrm{Ca}$, and $\mathrm{Fe}$. In contrast to Axelrod (1980; these proceedings), who include only $\mathrm{Fe}, \mathrm{Co}$ and $\mathrm{O}$, we do not calculate the line emission of $\mathrm{Fe}$ and $\mathrm{Co}$ in detail. This is reasonable for massive stars, where most of the emission is dominated by the lighter elements, but of course insufficient for Type Ia's. Neither do we include hydrogen, since we are primarily interested in the emission from the region where nuclear processing has taken place.

\section{GENERAL CONSIDERATIONS}

The most interesting question is the correspondence between the abundance structure of the supernova ejecta and the resulting optical emission observed (FC87). To illustrate this we show in Fig. 1 the emission per unit mass in the various lines as a function of the mass from the center for the Woosley and Weaver (1987) $8 \mathrm{M}_{\odot} \mathrm{He}$-core (main sequence mass $25 \mathrm{M}_{\odot}$ ), 300 days after the explosion. The expansion velocity of the 


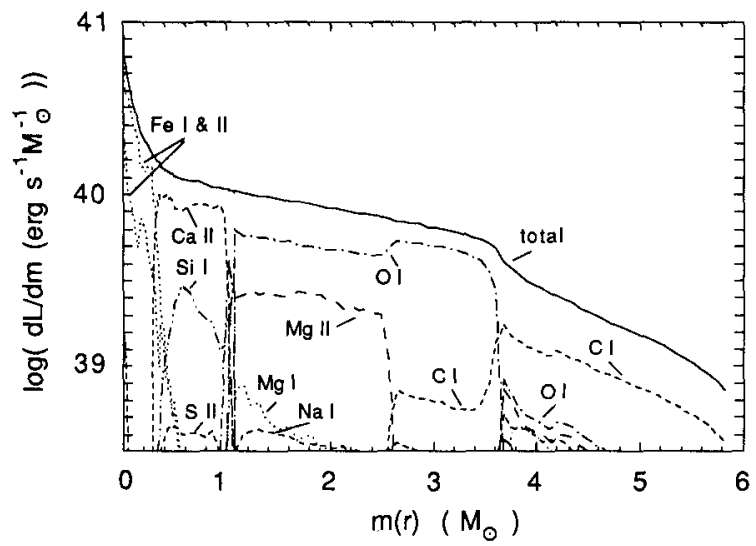

Fig 1. Luminosity per unit mass, $\mathrm{dL} / \mathrm{dm}$, of the most important lines 300 days after the explosion, for the Woosley and Weaver (1987) 8B model (ZAMS $25 \mathrm{M}_{\odot}$ ). The spectrum from this model is shown in Fig. 3.

core was $5200 \mathrm{~km} / \mathrm{s}$, and the total energy $3 \times 10^{51}$ ergs. This model is discussed further in Sect. 4. Comparing the figure with the abundance structure (Woosley and Weaver, 1987), we see that there is a rough overall correspondence between the nuclear burning shells and the emission from the various zones. However, a closer examination shows that it is extremely important to take the detailed atomic physics into account. This is evident for the Hemantle, where He does is not contribute much to the emission, which is instead dominated by the trace elements, mainly $C I$ and $O I$. The same thing can be seen in the inner part of the oxygen shell, where $\mathrm{Mg} \mathrm{I}, \mathrm{Mg}$ II and $\mathrm{Na}$ I lines are comparable to O I, even though the total abundances are down by factors of $\sim 25$ and $\sim 200$, respectively. Therefore, including the low abundance elements is necessary for a realistic comparison with observations. Neglect of this may severely overestimate the emission from the dominant elements.It is important to realize that the presence of strong $[O \mathrm{I}]$ lines in the spectrum does not mean that the density has to be less than the critical density, $\sim 10^{6} \mathrm{~cm}^{-3}$. While the thermalization cause the forbidden lines to radiate less efficiently compared to eg. the semi-forbidden, there are very few semi-forbidden or allowed transitions for neutral and singly ionized elements. This means that there are few channels other than the forbidden to do the cooling. As shown by the figure most of the elements lighter than iron can be studied from observations of optical and near-IR lines. The most important exceptions are $\mathrm{Ne}$ and $\mathrm{Ar}$, which have no lines in this region of the spectrum. There are, however, in the far-IR fine-structure transitions of these at $12.81 \mu$ ([Ne II]) and at $6.985 \mu$ ([Ar II]), which should become prominent especially at late epochs ( 450 days and later).

In general, the total emission from a particular burning zone is proportional to the $\gamma$-ray optical depth through the zone, $\Delta \tau_{\gamma}=\kappa_{\gamma} \int \rho(r)$ dr. The density structure is thus important for the relative line strengths. Fortunately, both this and the core velocity may be obtained directly from observations of the line profiles at late epochs. The velocity field has then relaxed to a $V \propto r$ law, and for an optically thin line the emission per volume, $j(r)$, is related to the intensity, $I(\varepsilon)$, of the line at the dimensionless frequency $\varepsilon$ by

$$
j(r=\varepsilon R)=\frac{1}{2 \pi \varepsilon R^{2}} \frac{d I(\varepsilon)}{d \varepsilon}
$$


Here $\varepsilon=\left(1-v / v_{0}\right) c / V_{0}$, and $v$ and $v_{0}$ are the frequency and the rest frequency of the line, respectively, $R$ the maximum radius of the supernova and $V_{0}$ the velocity at this point (ie. $\left.R / V_{0}=t\right)$. Therefore from the observed line profile one can determine the emissivity as a function of the radius $E R$, and thus the $\gamma$-ray input and density. If the energy input is dominated by $\gamma$-rays, the heating per volume is $\sim 0.03 \rho \mathrm{L}_{\gamma} /\left(4 \pi \mathrm{r}^{2}\right)$, where $\rho$ is the density and $\mathbf{r}$ the distance from the center. In the simple, but important, case where all the cooling is dominated by a single line we obtain the relation (since $r=\varepsilon R$ )

$$
\rho(r=\varepsilon R) \quad \propto \varepsilon \frac{d I(\varepsilon)}{d \varepsilon}
$$

Thus the shape of the line profile directly reflects the density distribution of the ejecta. It is, however, important to check whether the lines are really optically thin, since even the forbidden can have depths of the order of unity or larger. For [O I] $\lambda 6300$ we have $\tau=2$ (n) $O$ I) $\left./ 10^{9} \mathrm{~cm}^{-3}\right) \mathrm{t}_{\mathrm{yr}} \sim 1.1 \mathrm{M}_{\mathrm{c}}\left(\mathrm{V}_{\mathrm{c}} / 10^{3} \mathrm{~km} / \mathrm{s}\right)^{-3} \mathrm{t}_{\mathrm{yr}}{ }^{-2}$.

\section{TYPE Ib SUPERNOVAE}

The main characteristics of the Type I b supernovae are: 1) Occurrence close to $\mathrm{H} I I$ regions, 2) radio emission, presumably due to circumstellar interaction, 3) underluminous compared to Type Ia's, indicating a smaller mass of $56 \mathrm{Ni}, 4)$ after $\sim 200$ days the spectra are dominated by strong [O I] lines (see Panagia (1987) for a review). In Fig. 2 the beautiful spectrum of SN 1985f, obtained by Fillipenko and Sargent (1986) in March/April 1985, is shown. Unfortunately, for this supernova the exact time since the explosion is not known, but from a comparison with similar observations of SN 1983n Gaskell et al. (1986) argue that the epoch is $\sim 200-300$ days. Note the complete absence of any broad $\mathrm{H} \alpha$ line, immediately showing that the gas has undergone advanced nuclear processing. Although not yet settled

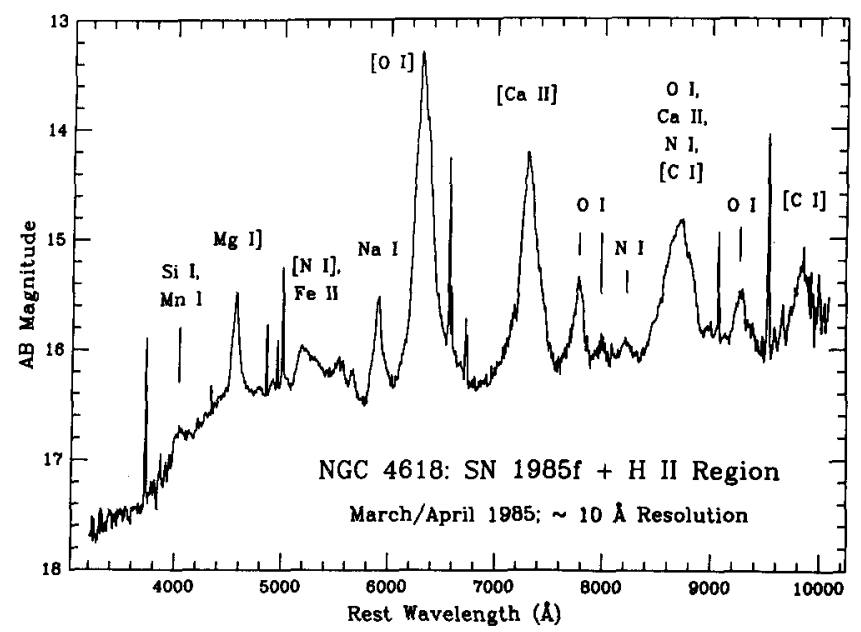

Fig. 2. Spectrum of SN 1985f in March/April 1985 from Fillipenko and Sargent (1986). Note the broad lines, the absence of a broad $\mathrm{H} \alpha$ line and the peaked profiles of the lines. 
(for a different view see Branch, this volume), most of these properties indicate a massive progenitor (Fillipenko and Sargent, 1985; Chevalier, 1986; Wheeler and Levreault, 1986;

Fransson, 1986). From a nebular analysis of the O I and O II lines, Begelman and Sarazin (1986) proposed that SN $1985 \mathrm{f}$ was the result of the explosion of a $\sim 50 \mathrm{M}_{\odot}$ Wolf-Rayet star, which had undergone a pair-instability collapse. Since the frequency of Ib's is comparable with the Type Ia's (Panagia, 1987), this origin is unlikely to apply for all Ib's. The main conclusion of their nebular analysis was that a minimum of $\sim 5 \mathrm{M}_{\odot}$ of oxygen was needed to explain the observations, making a very massive progenitor necessary. There are, however, several loop-holes in their argument. The most obvious are the uncertainties in the distance and reddening, which affect the [O I] $\lambda$ 6300-64 flux, and thus also the oxygen mass. Taking these into account, as little as $\sim 1 \mathrm{M}_{\odot}$ of oxygen is possible. Another complication is that the $O$ I and $O$ II zones in general do not coincide, meaning that the constraints from the $O I$ recombination lines are further relaxed. It is thus difficult to make any definite conclusions from this type of analysis, although it is probably difficult to escape the conclusion that at least $\sim 1 \mathrm{M}_{\odot}$ of oxygen is needed.

In addition to the oxygen lines, the late spectra of the $I$ b's also display emission lines of several other ions, like C I, Na I, Mg I, and Ca II (Fig. 2), from which additional constraints can be obtained. For realistic conclusions one must, however, calculate a selfconsistent model, with a density and abundance structure of the ejecta given by hydrodynamic calculations (or better, from observations of line profiles). As input models we have used the Woosley and Weaver (1987) models of exploding WR stars, without hydrogen envelope. Light curves for this type of models have been calculated by Ensman and Woosley (1986). Compared to the observed Type Ib light curves they, however, give too wide a peak. This may constrain the mass and energy severely for a WR origin. However, since the density distribution of these models do not reproduce the observed line profiles (see below), it is too early to make any definite conclusions. In this connection it should also be remembered that current models have difficulty in explaining the observed light curve for SN 1987a.

For a qualitative comparison we have calculated the spectrum of the exploded Woosley and Weaver (1987) $8 \mathrm{M}_{\odot}$ He core (ZAMS mass $25 \mathrm{M}_{\odot}$ ), model $8 \mathrm{~B}$, with a velocity of $\sim 5200 \mathrm{~km} / \mathrm{s}$ at the $\mathrm{O} / \mathrm{He}$ interface. In Fig. 3 the synthetic spectrum of this model is shown at 300 days. It should be stressed that there are very few free parameters in these models, except for the core mass, the ${ }^{56} \mathrm{Ni}$ mass and the core velocity, both set by the observations. Since especially elements with low ionization potentials, like $\mathrm{Na} \mathrm{I}, \mathrm{Mg}$ I and $\mathrm{Si}$ I, are sensitive to details, such as the photoionization by diffuse emission and the density structure, the spectra presented here should be regarded as preliminary results. Given this, there is a remarkably good qualitative agreement between the observations in Fig. 2 and Fig. 3 , both in terms of the lines present and their relative strengths. As noted by Fillipenko and Sargent, the identifications in Fig. 2 are uncertain due to the widths of the lines, and the feature at $4036 \AA$ is eg. consistent with [S I] $\lambda$ 4069-76 within the errors. We note that most of the emission in the line at $\sim 7296 \AA$ is due to the Ca II] $\lambda \lambda$ 7291-7324 lines, with only a small contribution from [O II] $\lambda \lambda 7320-30$. Also the wide feature at $8700 \AA$ is well modeled by a blend of O I 8446, Ca II $\lambda \lambda$ 8498-8662, and most important [C I] $\lambda$ 8727. The main discrepancy is in the $\mathrm{Na} I \lambda 5890$ strength, which is severely underestimated. This is, however, probably the most uncertain line, both in terms of the ionization balance and also in the total $\mathrm{Na}$ abundance in the models. The forbidden [Si I] lines at $1.099 \mu$ and $1.645 \mu$ are not in the range observed for this supernova, but the 1.6068-1.6455 $\mu$ lines were probably present 


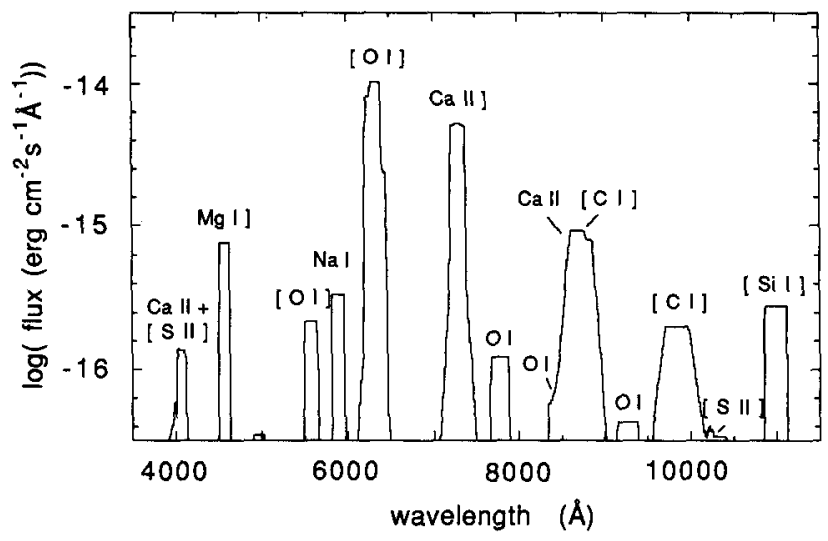

Fig. 3. Synthetic spectrum for the $25 \mathrm{M}_{\odot}\left(=8 \mathrm{M}_{\odot}\right.$ He-core) model 300 days after the explosion. The expansion velocity of the O-core was $\sim 5200 \mathrm{~km} / \mathrm{s}$. Note that several lines, eg [O I] $\lambda \lambda$ 6300-64 and Ca II $\lambda \lambda$ 8498-8662 + [C I] $\lambda 8727$ are blends. The large width of the [CI] $\lambda$ 9823-49 line is mainly due to the contribution of the high velocity He-mantle.

in the IR spectra of the Type Ib SN 1983n observed $\sim 1$ year after the explosion by Graham et al. (1986). These authors attributed the line to the [Fe II] 1.600-1.644-1.664-1.677 $\mu$ multiplet, but an equally likely interpretation, consistent with the expected strength, is due to the [Si I] line (Oliva, (1987); Fransson (1987)).

We have also tested WR models with smaller core masses, $4-6 \mathrm{M}_{\odot}$, but find considerably worse agreement, both in absolute and relative line strengths. Especially the $\mathrm{Ca}$ II / [O I] ratio is sensitive to the core mass, and may in fact require a somewhat higher mass than $25 \mathrm{M}_{\mathrm{o}}$ (see Sect. 5). Also an extreme white dwarf model, provided by Stan Woosley (priv. comm.) has been tested. In this the deflagration wave died out quickly, producing only $\sim 0.2 \mathrm{M}_{\odot}$ of ${ }^{56} \mathrm{Ni}$, leaving $\sim 0.5 \mathrm{M}_{\odot}$ of unburned $\mathrm{O}$ and $\sim 0.5 \mathrm{M}_{\odot}$ of $\mathrm{C}$, and $\sim 0.2 \mathrm{M}_{\odot}$ of other elements. The main problems with this is the small absolute strengths of the [O I] $\lambda \lambda$ 6300-64 lines, the far too large [C I] $\lambda \lambda$ 9823-49 / [O I] $\lambda \lambda$ 6300-64 ratio, and the large strengths of the [S I] lines. Most of the emission emerges as Fe emission. We thus find it unlikely that the Type Ib's can be explained by even extreme types of exploding white dwarf models.

The main problem with the WR models is that the line profiles in Fig. 3 are too flat, compared to the peaked profiles in Fig. 2. This is due to the shell structure of the core, due to the $56 \mathrm{Ni}$ heating, with nearly all the oxygen at one velocity. To estimate the required distribution of the ejecta, we have applied Eq. (2) to the blue wing of the [O I] $\lambda 6300$ line in the spectrum of SN 1985f (Fillipenko and Sargent, 1985), where the emission indeed was dominated by the [O I] $\lambda \lambda$ 6300-64 line. In Fig. 4 we show the density in dimensionless units as a function of the velocity, and thus radius ( $\mathrm{V} \propto \mathrm{r}$ ), indicating a much wider distribution in velocity than the models give. A possible explanation is that hydrodynamic instabilities during the first days may lead to mixing between different burning shells, and a smoothing of the distribution. Also, if clumps of the dense core material penetrate into the He-mantle and H-envelope, the line profiles of the metal lines will extend to higher velocities. Observations of Cas A (Chevalier and Kirshner, 1979) show evidence for this type of mixing. 


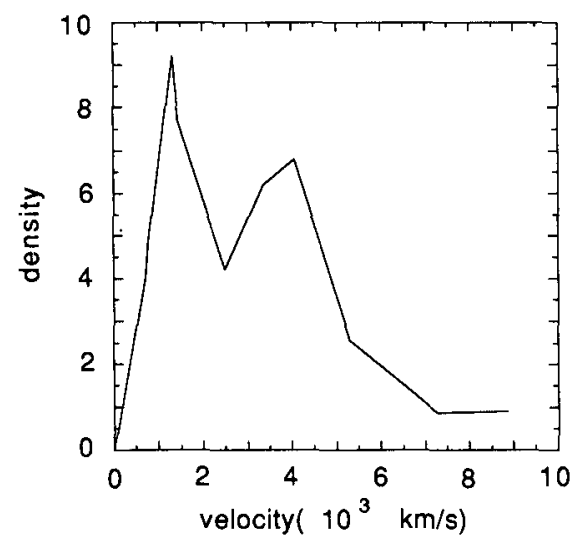

Fig. 4. Density as a function of expansion velocity $(\mathrm{V}(\mathrm{r}) \propto \mathrm{r}$ ) for the Type $\mathrm{Ib} \mathrm{SN} 1985 \mathrm{f}$, derived from the $[O \mathrm{I}] \lambda \lambda 6300$ line profile.

\section{TYPE II SUPERNOVAE AND SN 1987a}

Type II supernovae are thought to arise as the result of the explosion of stars more massive than $\sim 10 \mathrm{M}_{\odot}$. As we have already discussed the main difference between Type II's and the explosion of a WR star is the presence of a massive hydrogen envelope for the former. For the late spectrum of Type II's this results in a very strong $\mathrm{H} \alpha$ line, dominating the spectrum (see eg. the spectrum of SN 1979c by Branch et al., 1981). Uomoto and Kirshner (1986) have shown that for SN 1980k the luminosity of this line decayed with a rate close to the ${ }^{56} \mathrm{Co}$ decay time, as well as the total luminosity evolution observed by Barbon et al. (1982). This implies a $\gamma$-ray optical depth larger than unity. In that case one, however, expects the excited region to grow with the falling density, and the maximum velocity of the $\mathrm{H} \alpha$ line should increase. Since this is not observed, direct $\gamma$-ray excitation of the line is unlikely. The exact excitation is uncertain, but probably involves a combination of collisional excitation and ionizations by the Balmer continuum, followed by recombination. If the Balmer continuum flux follows the $\gamma$-ray input, as is likely, this may be reflected in the strength of $\mathrm{H} \alpha$. The large $\mathrm{H} \alpha / \mathrm{H} \beta$ ratio indicates a large optical depth in $\mathrm{H} \beta$, transforming these photons into $\mathrm{H} \alpha$ and $\mathrm{P} \alpha$.

For the understanding of the nucleosynthesis the $\mathrm{H} \alpha$ emission is of moderate importance. The expansion of the core is, however, sensitive to the presence of a hydrogen envelope. Because of the deceleration, the O-core velocity is only $1000 \mathrm{~km} / \mathrm{s}$ and that of the He-core $\sim 2500 \mathrm{~km} / \mathrm{s}$ (Woosley 1987). The lines are thus expected to be narrower and the $\gamma-$ ray optical depth higher compared to Type $\mathrm{Ib}$ 's, $\tau_{\gamma} \propto \mathrm{V}_{\mathrm{c}}{ }^{-2}$. Therefore, the $\gamma$-ray trapping is expected to be efficient for a longer time, and the light curves of both SN 1979c and SN $1980 \mathrm{k}$ showed no deviation from a pure exponential, more than a year after the explosion. Except for the $\mathrm{H} \alpha$ emission surprisingly little is known about the spectra of these at late phases. Recent observations by Fillipenko (1987) of SN 1986i, $\sim 9$ months after the explosion, however, show a number of strong emission lines of O I, $\mathrm{Ca}$ II and $\mathrm{Na}$ I. The widths of these 
lines indicate an origin in the core. As was seen in Fig. 1, there may, however, also be a strong contribution to these lines from the He-mantle. This supernova is also interesting, since its early spectrum resembles that of SN 1987a.

Obviously the late spectrum of SN 1987a is of great interest for the understanding of the nucleosynthesis, and I will here discuss some results, which attempt to show what kind of observations are relevant, as well as to indicate the kind of information one can obtain from this type of analysis (FC87; Fransson 1987). In FC87 we studied two different models, one $15 \mathrm{M}_{\odot}$ model with a core velocity of $\sim 1200 \mathrm{~km} / \mathrm{s}$, and one $25 \mathrm{M}_{\odot}$ model with core velocity of $2600 \mathrm{~km} / \mathrm{s}$. One interesting result was the increase in the [O I] $\lambda 6300-64 / \mathrm{Ca}$ II] $\lambda 7300$ ratio with increasing mass. Since both $\mathrm{O}$ I and $\mathrm{Ca}$ II are the dominant ionization stages, this ratio is probably fairly independent of the details of the ionization, in contrast to the $\mathrm{Mg} \mathrm{I}$ and $\mathrm{Na}$ I lines, and should mainly reflect the nucleosynthesis. The ratio does, however, depend on the amount of mixing of $\mathrm{Ca}$ and $\mathrm{O}$, which is uncertain in the explosion models. A mixing should also show up in the widths of the lines. Observations of these lines are therefore a useful probe of the nucleosynthesis.

As an example of this type of models, a calculation of a $20 \mathrm{M}_{\odot}$ (ZAMS) explosion model, specifically designed for SN 1987a (Woosley, 1987) has been done. Due to the $56 \mathrm{Ni}$ heating during the first days, most of the core mass is piled up in the high density peak outside the central ${ }^{56} \mathrm{Ni}$ bubble. This density distribution will give rise to a flat profile, similar to those in Fig. 3, but with a half width of $\sim 1000 \mathrm{~km} / \mathrm{s}$. High resolution observations can thus provide a badly needed test of the hydrodynamic calculations, as well as on the distribution of the individual elements. In Fig. 5 we show the spectrum in the optical and near-IR wavelength range one year after the explosion, and in Table 1 we give the line luminosities. This model is at the border line between the $\mathrm{Ca}$ II] dominated and [O I] dominated models, with [O I] $\lambda \lambda$ 6300-64 / Ca II] $\lambda 7300 \sim 0.9$

Table 1.

Luminosities after one year relative to [O I] $\lambda \lambda 6300-64$ of the strongest lines for the $20 \mathrm{M}_{\odot}$ model BF7 from Woosley (1987). L(6300-64) is the absolute luminosity of the [O I] $\lambda \lambda$ 6300-64 line, $\mathrm{V}_{\mathrm{c}}$ is the $\mathrm{O}$-core velocity, and $\mathrm{M}(56 \mathrm{Ni})$ the ${ }^{56} \mathrm{Ni}$ mass.

$\begin{array}{llll}\mathrm{M}(56 \mathrm{Ni})\left(\mathrm{M}_{\odot}\right) & 0.075 & \mathrm{~V}_{\mathrm{C}}(\mathrm{km} / \mathrm{s}) & 1200 \\ \mathrm{~L}(6300-64)(\mathrm{erg} / \mathrm{s}) & 2.1 \times 10^{39} & \end{array}$

\begin{tabular}{llllll}
\hline [C I] & 8727 & 0.64 & Mg II & 2800 & 0.74 \\
[C I] & $9823-49$ & 0.12 & [Si I] & 10991 & 0.49 \\
O I & 1356 & 0.04 & [Si I] & 16455 & 0.24 \\
[O I] & 5577 & 0.25 & [S II] & $4069-76$ & 0.07 \\
[O I] & $6300-64$ & 1.00 & [S II] & $10286-10371$ & 0.05 \\
$\mathrm{OI}$ & 7774 & 0.21 & [S I] & $25.25 \mu$ & 0.10 \\
[O I] & $63.15 \mu$ & 0.04 & [Ar II] & $6.985 \mu$ & 0.014 \\
[Ne II] & $12.81 \mu$ & 0.006 & Ca II & $3934-68$ & 0.17 \\
$\mathrm{Na} \mathrm{I}$ & $5890-96$ & 0.76 & Ca II] & $7291-7332$ & 0.91 \\
$\mathrm{Mg}$ I] & 4571 & 0.43 & Ca II & $8498-8662$ & 0.49 \\
\hline
\end{tabular}




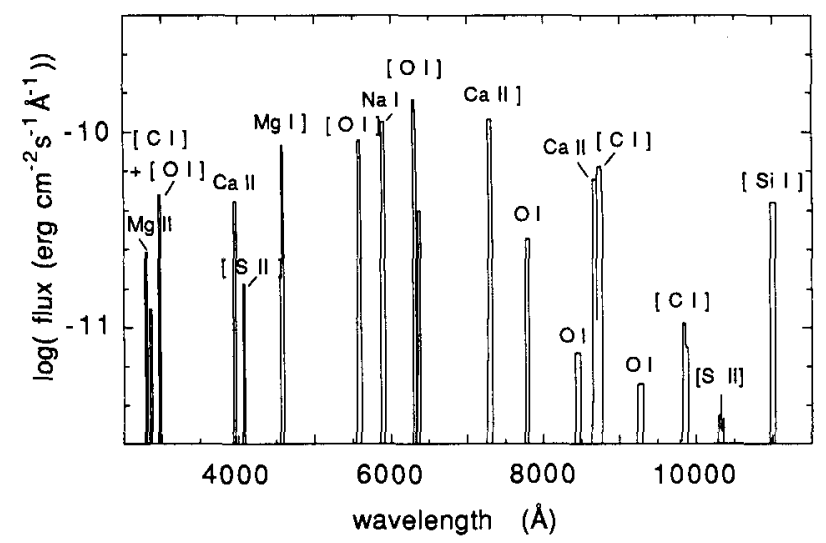

Fig. 5. Synthetic spectrum one year after the explosion of model BF7 from Woosley (1987), The ZAMS mass was $20 \mathrm{M}_{\odot}$ and the oxygen core velocity $\sim 1200 \mathrm{~km} / \mathrm{s}$.

Although the He-mantle was included in the calculations, there is very little trace of $\mathrm{He}$ in the spectrum, which is due to the low cooling efficiency of this element. Most of the absorbed energy is emitted as [C I] $\lambda \lambda$ 8727, 9824-50 lines. Also the [O I] and Ca II] lines may contribute to the cooling of the He-mantle, depending on the relative abundances of $\mathrm{C}, \mathrm{O}$ and $\mathrm{Ca}$ in the envelope. Lines from this region are expected to show a larger velocity width than lines coming solely from the core. Unless mixing is substantial, the iron lines are expected to come from the inner core. The emission from this region has not been calculated in detail, but the total Fe I-II emission is for both models less than $\sim 20 \%$ of the total. Mixing of the iron throughout the core may, however, increase this substantially.

When comparing these results with observations it should be kept in mind that the line strengths in some cases are sensitive to, for example, the density distribution of the ejecta. This is especially true for the $\mathrm{Na} \mathrm{I}, \mathrm{Mg} \mathrm{I}$, and [Si I] lines, for which the ionization is dominated by photoionization by diffuse UV emission, mainly from $\mathrm{O} I$ recombination lines. This is sensitive to both the density and to resonance line blocking in the UV. At epochs less than $\sim 450$ days the density in the core can be so high that forbidden lines like the $[\mathrm{O}$ I] and [Si I] lines are optically thick, resulting in P-Cygni type absorptions also for these lines.

The time dependence of the emission line luminosities is set by the decaying $\gamma$-ray input, proportional to $\tau_{\gamma} \mathrm{L}_{\gamma} \propto \mathrm{t}^{-2} \mathrm{e}^{-\mathrm{t} / 114 \mathrm{~d}}$. As discussed in detail in FC87 and Fransson (1987), this may lead to a thermal instability at $\sim 700$ days, due to a transition from cooling by optical and near-IR transitions to cooling by far-IR fine structure lines. Most of the emission then emerges as far-IR lines, like [O I] $63.15 \mu$, [Ne II] $12.81 \mu$, [Si I] $1.645 \mu$, [Si I] $68.49 \mu$, [S I] $25.25 \mu$, and [Ar II] $6.985 \mu$. These may be strong also at earlier epochs, and are thus important to monitor. They also have the advantage of being easy to model and should thus yield

The thermal instability results in a sudden drop of the temperature from $\sim 3500 \mathrm{~K}$ to less than $1000 \mathrm{~K}$. In the case of a clumpy density distribution the instability may set in earlier, and may also trigger the formation of dust in this metal rich environment. In this 
connection it is interesting that recent observations of SN 1987a have shown evidence of $\mathrm{CO}$ in the ejecta (McGregor and Hyland, 1987). Preliminary calculations show that this requires the temperature to be less than $\sim 4000 \mathrm{~K}$, and the electron fraction to be less than $\sim 0.1$. Since the lines are fairly broad, they are, however, likely to be formed in the hydrogen envelope. At later stages molecule formation may also occur in the core regions, and be the first sign of dust condensation.

Finally, the presence of a pulsar may be important after about two years, when the radioactivity has decayed. A pulsar will be surrounded by a synchrotron nebula (a plerion), emitting ionizing radiation. The ejecta will thus be gradually ionized, as they expand. In contrast to the $\gamma$-ray heated case most of the absorbed energy is expected to be emitted as lines of highly ionized elements in the UV. The ionization of the envelope will also cause free-free absorption of the radio emission from the plerion, making a detection of this difficult during the first few years. For more details I refer to FC87 and Fransson (1987). Chevalier (1987) has proposed that the optical emission from SN 1986j (Rupen et. al., 1987) may be the result of pulsar excitation. Although the age of the supernova is not known, Chevalier argues from the evolution of the radio emission that it is $\sim 4$ years. The fact that the optical spectrum shows a number of strong He I lines with high excitation potentials may be consistent with photoionization by a central hard continuum source. Also the low velocities, FWHM $1000 \mathrm{~km} / \mathrm{s}$, argues for emission from the core. The modelling of this is, however, very sensitive to mixing, filamentation etc., known to be important for the Crab.

\section{CONCLUSIONS}

The discussion in this paper shows that we in the future can expect the understanding of the stellar nucleosynthesis from observations of supernovae to be considerably improved. Most of the physics in connection with the thermalization of the $\gamma$ rays is well understood, as well as most of the atomic data going into the calculations. There are, however, in this area some uncertain processes, most importantly the charge transfer reactions between the various ions, like O II + Na I $\sim \mathrm{O} \mathrm{I}+\mathrm{Na}$ II. Also the ionization of the trace elements, $\mathrm{Na} \mathrm{I}, \mathrm{Mg}$ I and $\mathrm{Si} I$, may be sensitive to the treatment of the $\mathrm{UV}$ radiation field. However, these problems are likely to be solved in the near future. Therefore, from a given explosion model of the density and abundance structure one can predict what the late spectrum should be, and compare this with the observations. Especially the line profiles are important, since they provide a test of the probably most uncertain part of the explosion calculations.

If the late spectra of $\mathrm{SN}$ 1987a do not correspond to the model predictions this is hardly surprising. However, using these observations I think that with our current knowledge of the spectral formation we can learn a lot about the structure of the supernova and of nucleosynthesis. It should also be remembered that SN 1987a is only one single event, and that it is necessary to test the models for a wide range of progenitors. It is thus important to get good spectral information about other more distant supernovae. In this respect we can expect much from the future, since the observational requirements are already within our capabilities. 
Acknowledgements: Most of this work has been done in collaboration with Roger Chevalier. I am also grateful to Claes-Ingvar Björnsson for reading the manuscript and for discussions, to Stan Woosley for providing the models of SN 1987a, and to Alexei Fillipenko for the spectra of SN $1985 \mathrm{f}$.

\section{REFERENCES}

Axelrod, T.S.: 1980, Ph.D. thesis, Univ. of California, Santa Cruz.

Barbon, R., Ciatti, F., and Rosino, L.: 1982, Astron. Astrophys. 116, 35.

Barbon, R., Cappelaro, E., and Turatto, M.: 1984, Astr. Ap. 135, 27.

Begelman, M.C., and Sarazin, C.L.: 1986, Astrophys. J. (Letters) 302, L59.

Branch, D., Falk, S.W., McCall, M.L., Rybski, P., Uomoto, A.K., Wheeler, J.C., and Wills, B.J.: 1981, Astrophys. J. 244, 780.

Branch, D., Doggett, J.B., Nomoto, K., and Thielemann, F.-K.: 1985, Astrophys. J. 294, 619.

Chevalier, R.A.: 1986, in Highlights of Astronomy, Ed. J.P. Swings, (D. Reidel: Dordrecht) p. 599.

Chevalier, R.A.: 1987, Nature, 329, 611.

Chevalier, R.A., and Kirshner, R.P.: 1979, Ap. J. 233, 154.

Chiosi, C., and Maeder, A.: 1986, Ann. Rev. Astron. Astrophys. 24, 329.

Ensman, L.M., and Woosley, S.E. : 1986, B.A.A.S. 18, 963.

Fillipenko, A.V.: 1987, in Proc. of the 4th George Mason Conference on SN 1987A, ed. M. Kafatos, in press.

Fillipenko, A.V., and Sargent, W.L.W.:1985, Nature, 316, 407.

Fillipenko, A.V., and Sargent, W.L.W.:1986, Astron. J. 91, 692.

Fransson, C.: 1986, in Highlights of Astronomy, Ed. J.P. Swings, (D. Reidel: Dordrecht) p. 611.

Fransson, C.: 1987, in ESO Workshop on SN 1987A, Ed. J.I. Danziger, in press.

Fransson, C., and Chevalier, R.A.: 1987, Ap. J. (Letters), Nov. 1 1987. (FC87)

Gaskell, C.M., Cappellaro, E., Dinerstein, H.L., Garnett, D.R., Harkness, R.P. and Wheeler, J.C.: 1986, Astrophys. J. (Letters) 306, L77.

Graham, J.R., Meikle, P.S., Allen, D.A., Longmore, A.J., and Williams, P.M.: 1986, M.N.R.A.S. 218, 93.

Itoh, H. , and Nomoto, K.: 1987, in X-Ray Astrophysics, Eds. A.C. Fabian and C.R. Canizares, in press.

McGregor, P.J., and Hyland, A.R.: 1987, I.A.U. Circ. No. 4486.

Meyerott, R.E.: 1980, Astrophys. J. 239, 257.

Nomoto, K., Shigeyama, T., and Hashimoto, M.: 1987, in ESO Workshop on SN 1987A, Ed. J.I. Danziger, in press.

Oliva, E.: 1987, Astrophys. J. (Letters) 321, L45.

Panagia, N.: 1987, in High Energy Phenomena Around Collapsed Stars, Ed. F. Pacini, Reidel (Dordrecht), p.33.

Raymond, J. C.: 1984, Ann. Rev. Astron. Astrophys. 22, 75.

Rupen, M.P., van Gorkom, J.H., Knapp, G.R., Gunn, J.E., and Schneider, D.P.: 1987, Astron. J. 94,61 .

Uomoto, A., and Kirshner, R.P.: 1986, Astrophys. J. 308, 685.

Weaver, T.A., and Woosley, S.E.: 1980, in Supernova Spectra, ed. R. Meyerott and G.H.

Gillespie, American Institute of Physics, p. 15.

Wheeler, J.C., and Levreault, R.: 1985, Ap. J. (Letters), 294 , L17.

Woosley, S.E.: 1987, in prep. for Ap. J.

Woosley, S.E., and Weaver, T.A.: 1987, preprint. 